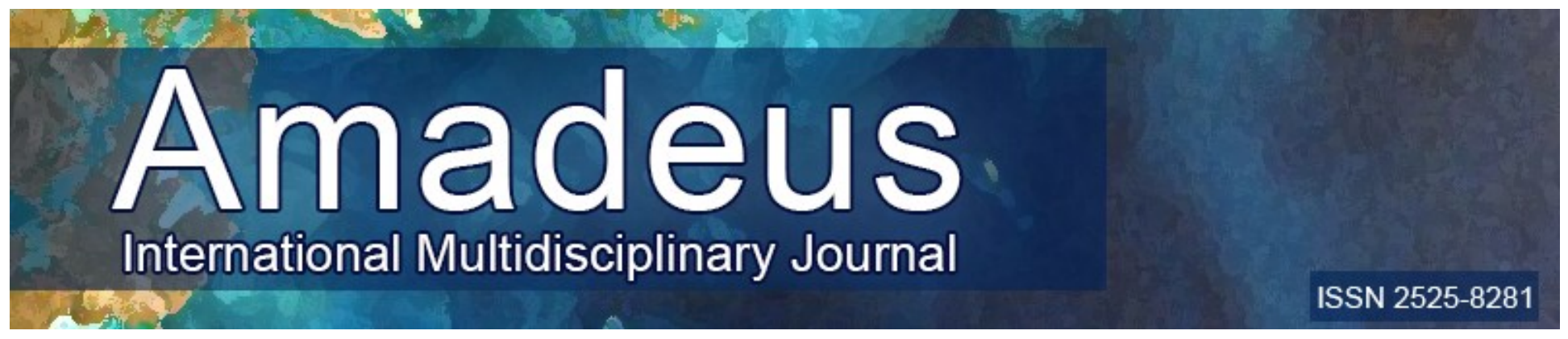

DOI: $10.14295 /$ AIMJ.V6I11.169

\title{
Facial reconstruction with extended supraclavicular flap after necrotizing fasciitis: Case report
}

\author{
Kaio Danilo Leite da Silva Rocha ${ }^{l}$, \\ Victor Hugo Melo Carvalho ${ }^{2}$, \\ Pedro Ivo Martins Cidade, \\ Allan Sávio Soares Macedo ${ }^{4}$, \\ Maria Isabel Fernandes Peixoto Furtado, \\ Livia Carvalho Bezerra de Brito Vieira ${ }^{6}$ \\ Francisco Felippe de Araújo Rolim ${ }^{7}$
}

Abstract: This case report describes a clinical case of face necrotizing fasciitis submitted to surgical treatment for facial reconstruction using an expanded flap. Infectious control of the lesion was performed with broad spectrum antibiotics and surgical debridement of the entire necrotic tissue, followed by reconstructive surgery, with preparation and transfer of the supraclavicular skin flap to the affected. This report highlights a rare case and emphasizes the importance of early diagnosis, the surgical approach carried out quickly and the success of repair using the autonomous supraclavicular flap. The expanded supraclavicular flap is an excellent option for facial reconstruction in patients in whom extensive areas of skin have been removed, as they present good vascularization and an adequate aesthetic appearance in the postoperative period.

Keywords: Autonomous flaps, facial reconstruction, supraclavicular flap, reconstructive surgery.

Resumo: Este relato de caso descreve um caso clínico de fasceíte necrotizante de face submetida a tratamento cirúrgico para reconstrução facial utilizando retalho supraclavicular estendido. Uma das abordagens para reconstrução facial é a utilização de retalho expandido, autonomizado. Realizou-se o controle infeccioso da lesão com antibioticoterapia ampla e desbridamentos cirúrgicos de todo tecido necrótico, procede-se a cirurgia reparadora, primeiramente com preparo do retalho e posterior transferência de pele supraclavicular estendida para o local afetado. Este relato evidencia um caso raro e enfatiza a importância do diagnóstico precoce, da abordagem cirúrgica realizada com brevidade e do sucesso com reparação utilizando o retalho supraclavicular autonomizado. O retalho supraclavicular expandido é uma excelente opção para reconstrução da face em pacientes em que foram removidas extensas áreas de pele, pois apresentaram boa vascularização e aspecto estético adequado no pós-operatório.

Palavras-chave: Retalhos autonomizados, reconstrução facial, retalho supraclavicular, cirurgia reconstrutiva, retalho cirúrgico.

${ }^{1}$ Resident in General Surgery at Hospital Regional do Cariri (HRC), Juazeiro do Norte, Ceará, Brazil.

${ }^{2}$ Resident in General Surgery at Hospital Regional do Cariri (HRC), Juazeiro do Norte, Ceará, Brazil.

${ }^{3}$ Resident in General Surgery at Hospital Regional do Cariri (HRC), Juazeiro do Norte, Ceará, Brazil.

${ }^{4}$ Resident in General Surgery at Hospital Regional do Cariri (HRC), Juazeiro do Norte, Ceará, Brazil.

${ }^{5}$ Medical Student at the Estácio de Juazeiro do Norte Faculty of Medicine (FMJ/IDOMED),

Juazeiro do Norte, Ceará, Brazil. Email: isabelp2111@gmail.com

${ }^{6}$ Plastic Surgeon at Hospital Regional do Cariri, Juazeiro do Norte, Ceará, Brazil.

${ }^{7}$ Digestive System Surgeon at Hospital Regional do Cariri, Juazeiro do Norte, Ceará, Brazil. 


\section{Introdução}

A fasceíte necrotizante (FN) é uma infecção rara e grave, caracterizada por necrose extensa e rapidamente progressiva. Ela acomete o tecido celular subcutâneo e a fáscia muscular ${ }^{14}$. A FN, que acomete a região de cabeça e pescoço, é uma infecção rara, que se espalha rapidamente, pelos tecidos moles, de origem polimicrobiana $^{15}$.

A reconstrução facial nos defeitos extensos é desafiadora; a primeira escolha são retalhos; de preferência com espessura, coloração parecida com a pele da face. Nesse contexto a utilização de retalho supraclavicular tem sido uma excelente opção, pois apresenta facilidade de retirada, mínima morbidade da área doadora e ausência de anastomose microvascular ${ }^{1}$. A expansão prévia de retalhos, tanto locais, como à distância, permite diminuir a morbidade da área doadora e fornecer tecido em quantidade e qualidade para a correção de extensos defeitos ${ }^{2,3}$ além disso, também assegura entre outras vantagens uma melhor vascularização do retalho, uma vez que a cápsula formada junto à pele expandida apresenta uma ampla rede de novos vasos ${ }^{4,5}$.

Uma reconstrução ideal fornece um retalho flexível e que pode substituir a pele da face com uma boa combinação de cor, textura e ser capaz de cobrir tanto pele mais delicada quanto defeitos profundos $^{1}$, além de garantir qualidade em relação à vascularização do tecido doado. Dentre as principais vantagens, podem ser destacados fatores relacionados à cicatriz de área doadora inconspícua, técnica simples e reprodutível, tempo cirúrgico curto, uso em pacientes com diversas comorbidades, retalho fino e glabro, cor semelhante da vizinhança a ser reconstruída ${ }^{6,7,8,9,10,11,12,13}$. Apresentamos um caso em que a paciente foi submetida à reconstrução facial utilizando tecido supraclavicular pré-estendido pós fasceíte necrotizante na face. 


\section{Relato de Caso}

Trata-se de paciente do sexo feminino, 45 anos, admitida no Hospital Regional do Cariri (Ceará) em 19/02/2020, proveniente de hospital de pequeno porte no interior do estado onde estava tratando sem sucesso lesão em face e pescoço secundária a abscesso odontogênico diagnosticado previamente.

$\mathrm{Na}$ admissão paciente relatava que o quadro iniciou por odontalgia em arcada dentária inferior direita associada a edema, rubor e dor intensa em hemiface e região cervical lateral direita há aproximadamente 15 dias, com piora progressiva apesar de tentativas de tratamento no hospital de origem. De comorbidades relata tabagismo e nega doenças crônicas, uso regular de medicamentos, cirurgias prévias ou alergias.

Ao exame, foi evidenciado a presença de extensa área de processo inflamatório tecidual com necrose em regiões bucinadora, parotidea, masseteriana, mandibular e cervical lateral direitas, com hiperemia na transição cérvico-torácica. Cavidade oral com limitação de abertura bucal e presença de área de necrose gengival a direita (Figura 1). 


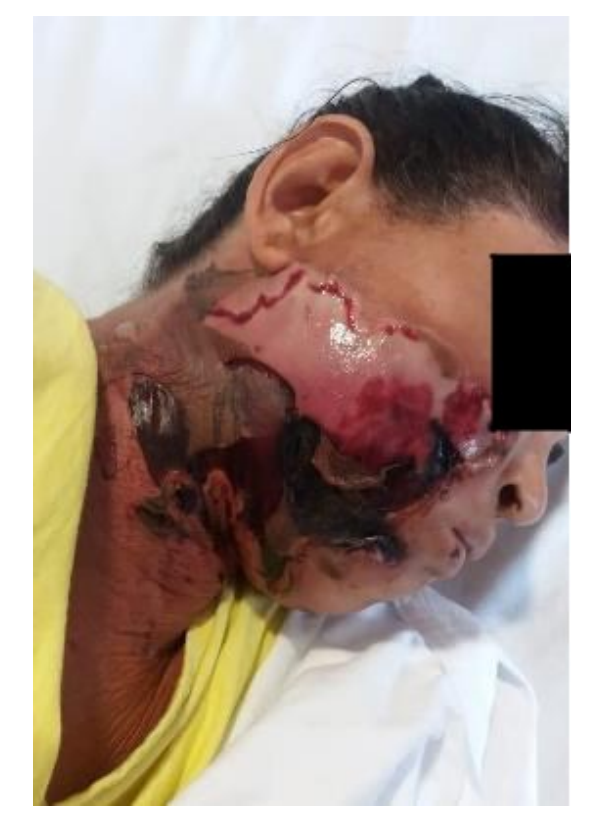

Figura 1. Lesão cérvico-facial à admissão.

Aventada hipótese de fasceíte necrotizante e iniciado terapêutica antimicrobiana ampla (Amicacina e Oxacilina) e investigação com exames complementares: tomografia de face e pescoço: extensa área de infiltrado infeccioso/inflamatório em região paramandibular direita sem coleções passíveis de drenagem; hemograma infecioso com desvio à esquerda, sem anemia e com plaquetopenia (hemoglobina: $13 \mathrm{~g} / \mathrm{dl}$, hematócrito: 42\%, leucócitos: 59.000, bastonetes: $11 \%$, plaquetas: 85.000 ); função renal normal.

Após 14 dias de antibioticoterapia evoluiu com regressão importante do processo inflamatório-infeccioso e delimitação de grande área de necrose cervicofacial direita ( $12 \mathrm{~cm}$ de diâmetro), quando foi submetida ao primeiro desbridamento cirúrgico no dia 03/03/2020 (Figuras 2 e 3). 


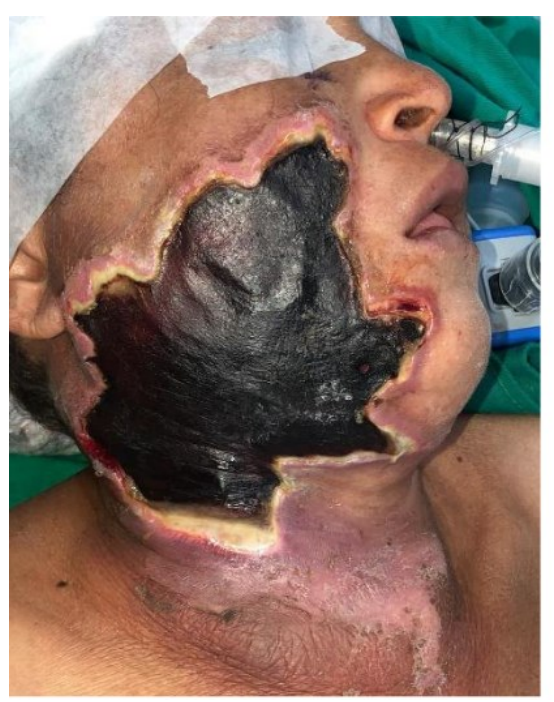

Figuras 2 e 3. Área de necrose delimitada. Aspecto pós primeiro desbridamento cirúrgico.

No $7^{\circ} \mathrm{DPO}$ paciente evoluiu com ferimento cervicofacial extenso à direita, envolvendo regiões bucomandibular, pré-auricular e cervical ântero-lateral com presença de granulação discreta, áreas de tecido desvitalizado, exposição óssea de mandíbula e fístula orofacial em região bucal. Apresentou novos picos febris altos $\left(41,1^{\circ} \mathrm{C}\right)$, piora infecciosa clínico-laboratorial sendo submetida a novo desbridamento cirúrgico e oclusão por meio de curativo com papaína, escalonado antibioticoterapia com meropenem e metronidazol orientadas por culturas, traqueostomizada, passagem de sondagem nasoenteral e internamento em UTI (FIGURA 4). 


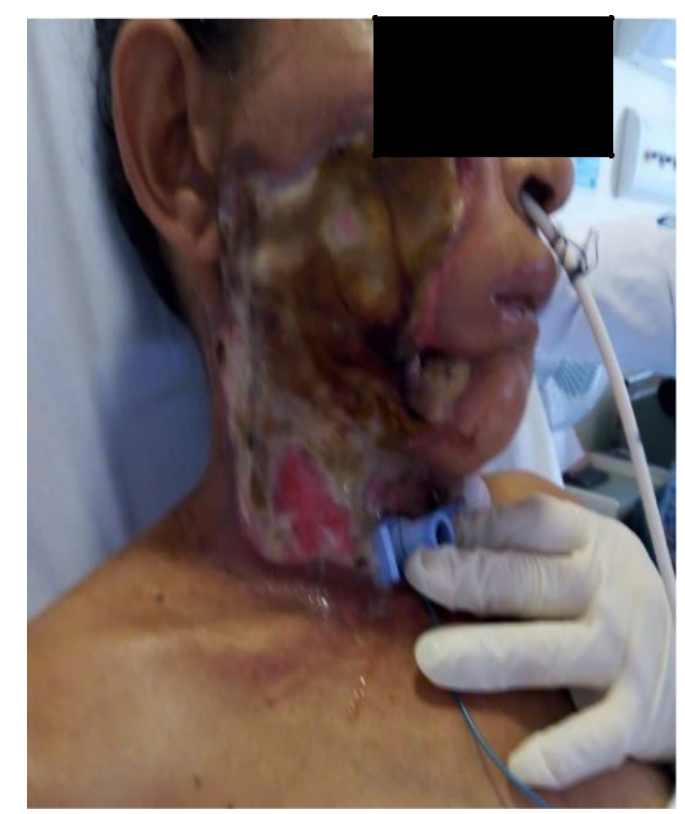

Figura 4. Evolução pós-operatória do primeiro desbridamento.

Após quatro dias seguiu em resolução do quadro infeccioso, recebeu alta da UTI para enfermaria onde permaneceu realizando tratamento com antibióticos e curativos diários (Figura 5).

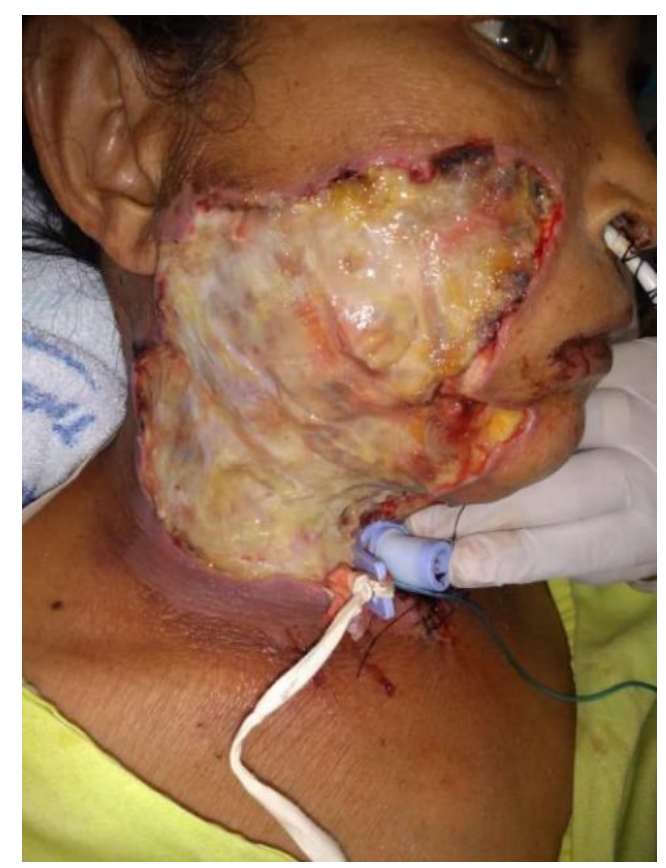

Figura 5. Evolução pós-operatória do segundo desbridamento.

Após 30 dias de tratamento paciente evoluiu com controle infeccioso, granulação da ferida, fístula orofacial e discreta área de fibrina e tecido necrótico 
central (Figura 6).

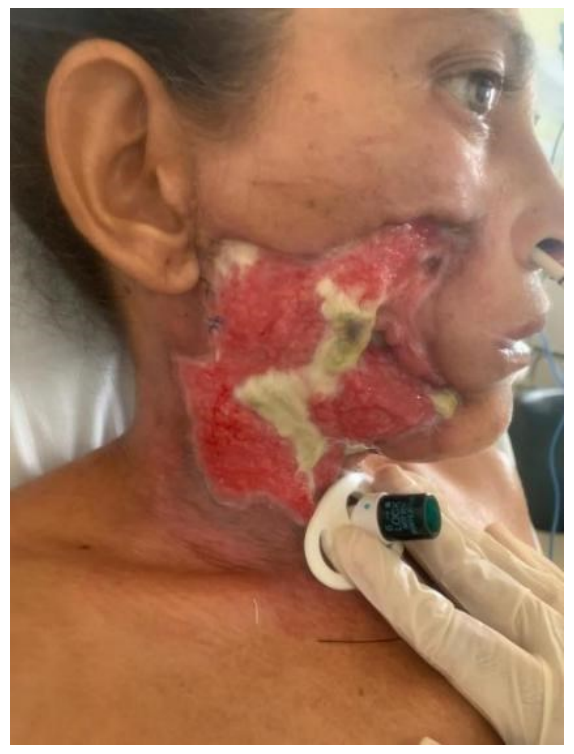

Figura 6. Evolução pós-operatória do primeiro desbridamento.

Optou-se então por iniciar o tratamento reparador, e a opção pela extensão da área a ser reparada, foi pelo retalho supraclavicular. Em 07/04/2020 foi realizado o primeiro procedimento para reconstrução: preparo de retalho da região supraclavicular para reconstrução da face da paciente. Iniciado com desbridamento de bordas de ferida cervicofacial, reposicionamento de mucosa oral com cobertura de fístula orofacial (utilizando Vicryl ${ }^{\circledR}$ 4.0) e a autonomização de retalho supraclavicular para proporcionar um retalho espandido, uma vez que a paciente é tabagista (Figura 7). 


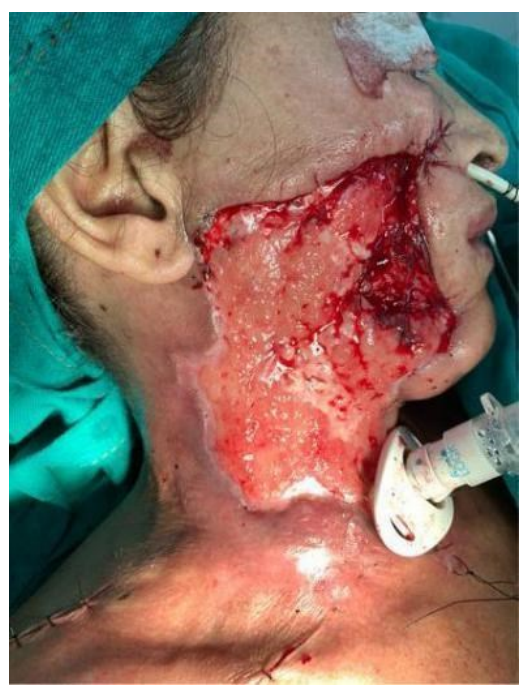

Figura 7. Primeira etapa da cirurgia reparadora - correção da fístura orofacial, debridamento da ferida e autonomização do retalho supraclavicular.

No $14^{\circ}$ DPO, a paciente evoluiu com boa autonomização do retalho supraclavicular e com boa granulação da ferida para recebimento do retalho reparador (Figura 8).

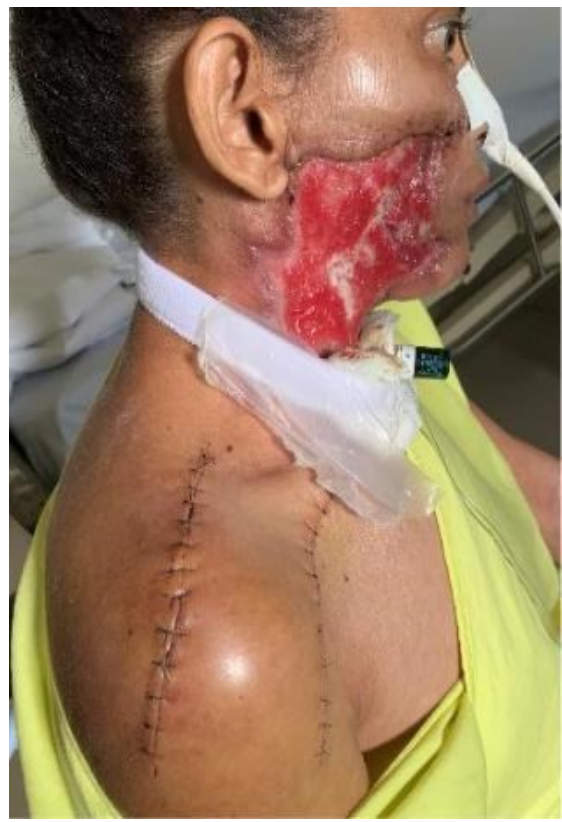

Figura 8. $14^{\circ} \mathrm{DPO}$ - correção da fístura orofacial, debridamento da ferida e autonomização do retalho supraclavicular para proporcionar um retalho expandido. 
Procedeu-se então a segunda etapa da cirurgia reparadora, iniciando pelo levantamento do retalho supraclavicular de lateral para medial, que apresentou boa perfusão distal, desepidermização cervical distal e posicionamento do pedículo do retalho seguido por acomodação do retalho na área cruenta, fixando-o com fio de nylon 4.0. Realizado amplo descolamento da área doadora em ombro direito e após fechamento por planos com Vicryl® 2.0 em subcutâneo e Nylon 2.0 em pele. Devido a extensão do descolamento, optado por drenagem com Portovac ${ }^{\circ} 4.8 \mathrm{e}$ curativo oclusivo. Optado, ainda por profilaxia estendida com Cefalotina (Figura 9).

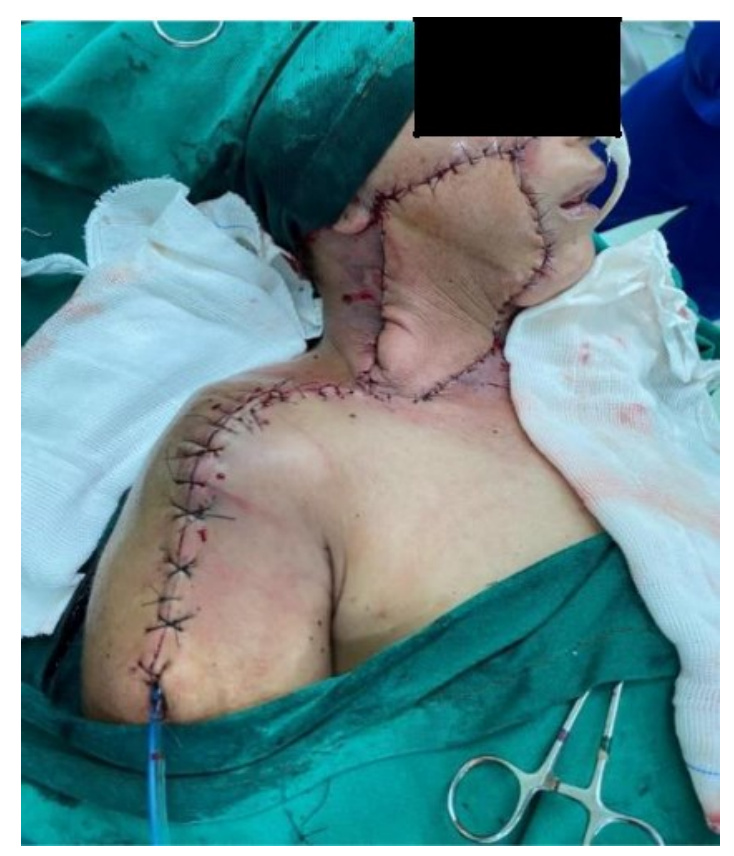

Figura 9. Segunda etapa da cirurgia reparadora - reconstrução cervicofacial com retalho supraclavicular estendido.

No $7^{\circ}$ DPO, a paciente evoluiu com boa perfusão do retalho, discreta epidermólise em área doadora e concluiu antibioticoterapia. Foi solicitado acompanhamento conjunto com fonoterapeuta para início de dieta mista (Figura $10)$. 


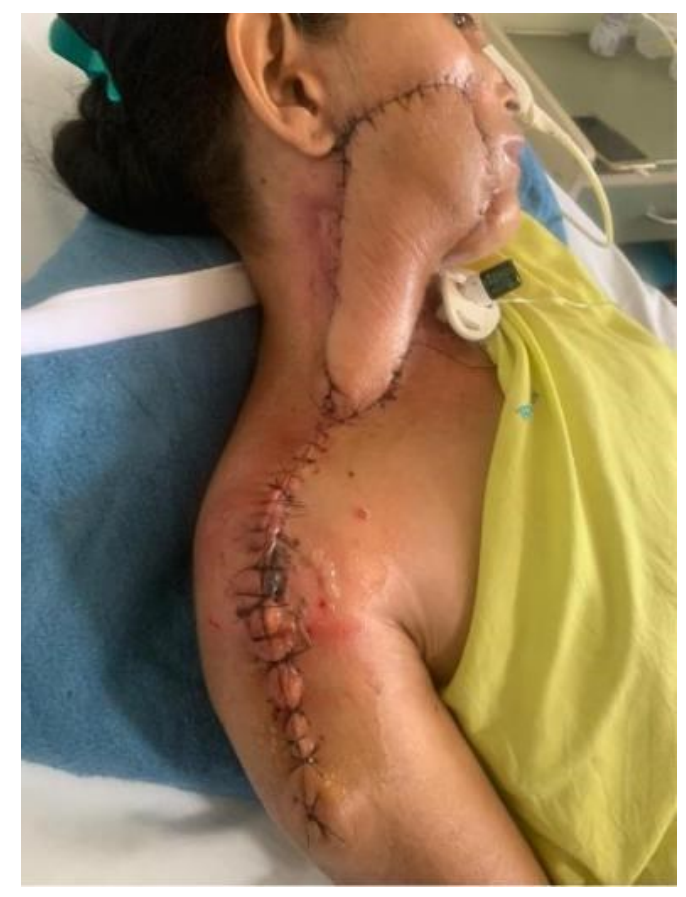

Figura 10. $7^{\circ}$ DPO de reconstrução cervico-facial com retalho supraclavicular estendido.

No $10^{\circ}$ dia pós-operatório recebeu alta hospitalar em com orientações de curativos diários sem compressão local e de retorno em 15 dias ao ambulatório de cirurgia plástica para acompanhamento (Figura 11).

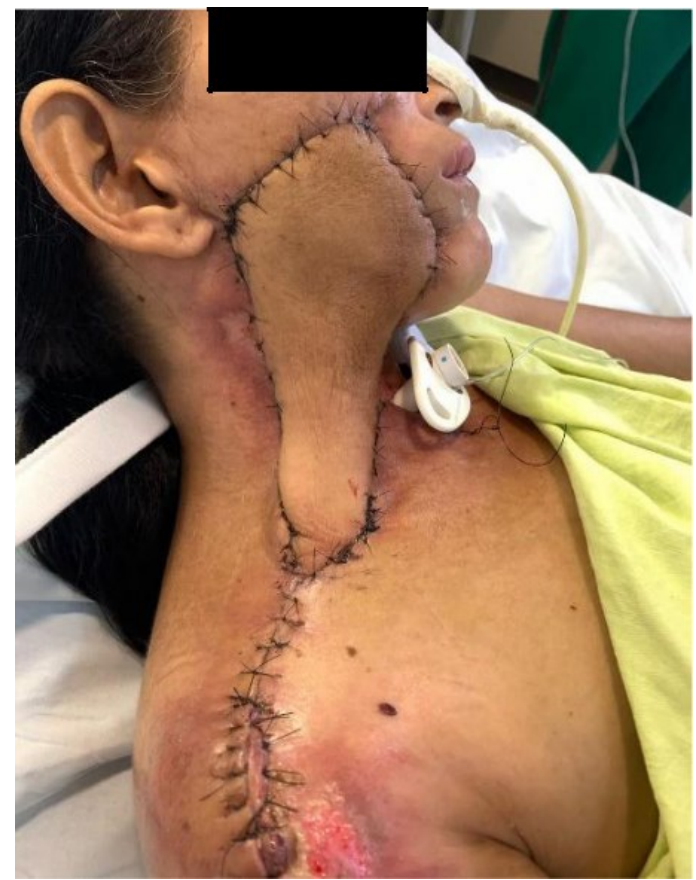


Figura 11. $10^{\circ}$ DPO de reconstrução cervico-facial com retalho supraclavicular estendido.

No $28^{\circ} \mathrm{DPO}$, a paciente retornou sem queixas, relatou que já se alimentava por conta própria sem engasgos. Ao exame físico foi avaliado positivamente o aspecto do retalho, área doadora com boa cicatrização, abertura bucal satisfatória, porém limitada à direita, mucosa oral íntegra e sem identificação de áreas cruentas. Retirados os pontos, encaminhada aos serviços de fonoaudiologia e nutrição para desmame da nutrição enteral e à cirurgia de cabeça e pescoço para avaliar a decanulação de traqueostomia. Orientado retorno ao setor de cirurgia plástica em seis meses para seguimento.

Decorridos sete meses da cirurgia, a paciente retornou ao ambulatório de cirurgia plástica do serviço, sem queixas relacionadas ao retalho e boa cicatrização da área doadora, aceitação da dieta por via oral. Ao exame físico, as cicatrizes tinham aspecto plano, hipocromicas, retalho com bom aspecto, sem sinais de fistula salivar, abertura bucal satisfatória. Recebendo alta da cirurgia plástica nesta data (Figuras 12 e 13).
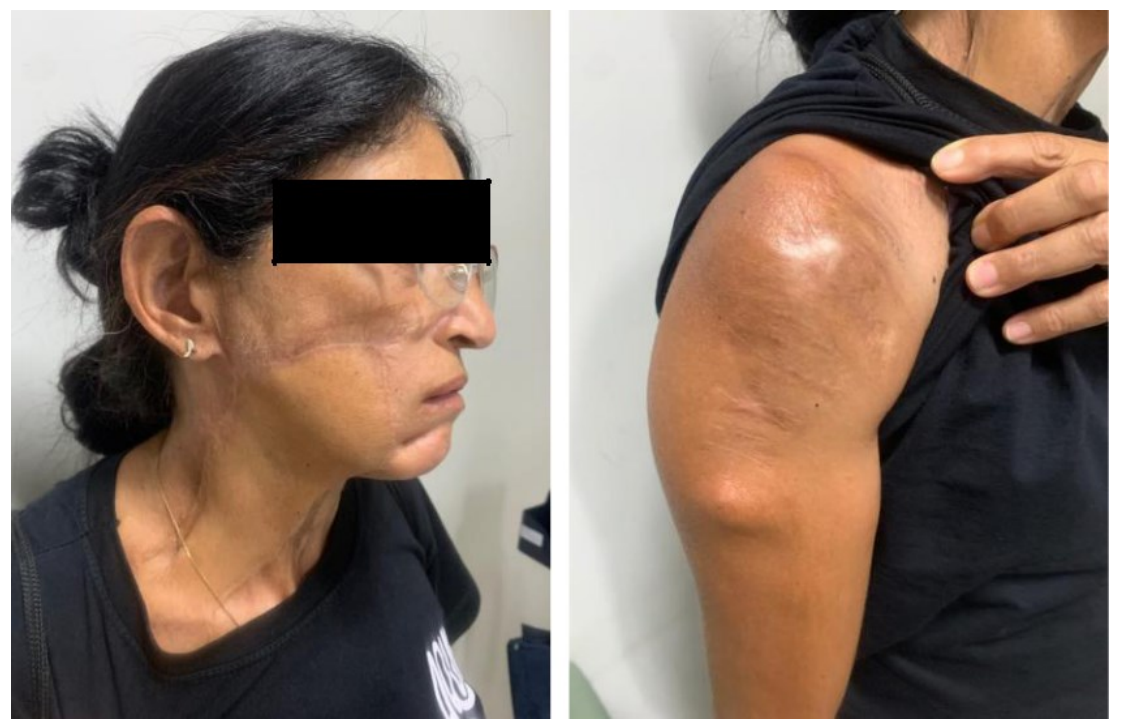

Figuras 12 e 13. Pós cirurgico de 7 meses de reconstrução da face. 12. 
retalho da face 13. área doadora.

\section{Discussão}

Fasceíte necrotizante apresenta características como invasão tecidual associada a necrose, manifestações sistêmicas sépticas graves, evidenciando a importância do pronto atendimento do paciente. Apesar de considerada uma doença rara, é uma infecção bastante grave de progressão rápida, e na face, a infecção se espalha ao longo do sistema músculo-aponeurótico superficial, um plano fascial contíguo com os músculos da expressão facial e se estende do músculo frontal ao platisma $^{14}$. O tratamento desses casos demandam uma terapêutica intra-hospitalar, inicialmente é indicada a antibioticoterapia endovenosa ampla e tratamento cirúrgico por desbridamento das regiões afetadas, para evitar evolução mais agressiva da doença ${ }^{15}$.

No caso relatado, a paciente foi submetida a tratamento cirúrgico de urgência e antibioticoterapia. Como tratamento primário, a paciente foi submetida à desbridamento de tecido desvitalizado, e devido à reincidência de infecção, a conduta foi a realização de novo desbridamento e posterior reconstrução da área. No caso relatado, o procedimento para reconstrução da face da paciente ocorreu por cobertura com retalho supraclavicular autonomizado. Colletti e colaboradores ${ }^{8}$ apresentaram um estudo em que relatam a utilização bem-sucedida de retalhos supraclavicular para estruturar um defeito de espessura na bochecha de pacientes com necrose na região cervicofacial.

O campo da cirurgia plástica reconstrutiva está em desenvolvimento contínuo, visando a qualidade de vida dos pacientes e aprimorar técnicas. A utilização do retalho supraclavicular tem recebido atenção dentre as técnicas cirúrgicas e se popularizou após Pallua et $\mathrm{al}^{16}$ ter demonstrado que era clinicamente 
segura. Garante um funcionamento adequado de irrigação sanguínea e reconstrução estética ocasionando morbidade mínima da área doadora, cor e textura aceitáveis correspondentes a pele facial e cervical, especialmente na região inferior do rosto, pescoço, e região da parede torácica superior, pode ser elevada $6,7,8,9,10,13,17$. Além disso, é considerando que o procedimento para retalhos loco-regionais é realizado de maneira mais rápida e são viáveis também em centros onde não há microcirurgia ${ }^{8,18}$.

A autonomização foi realizada na primeira sessão cirúrgica reparadora, após o controle infeccioso, para que posteriormente fosse feita a rotação do retalho na área receptadora. O retalho supraclavicular pode ser pré-expandido para aumentar o tamanho de tecido a ser doado, e é posteriormente transferido como uma ilha ou como uma aba peninsular, que precisa ser dividido e inserido alguns dias depois ${ }^{19,20,21,22,23}$. O intervalo e o volume de expansão podem variar

por região e tipo de ferida. No caso descrito, não foi utilizado expansores de pele e sim a autonomização do retalho com incisões previas seguidas de suturas demarcado o retalho a fim de autonomizar a área doadora e possibilitar resultados semelhantes e o procedimento transcorreu sem complicações.

Outro desafio relacionado à reconstrução facial é alcançar cobertura adequada de tecidos moles sem colocar tensão em estruturas como lábios ou pálpebras, sobretudo em se tratando de reconstrução em áreas que sofreram grande perda tecidual ${ }^{5,9}$ pode haver algum grau de cicatriz ou contratura, como às vezes é visto na reconstrução vestibular com este retalho ${ }^{10}$. A paciente em questão apresentou, após o $28^{\circ}$ dia de reconstrução, abertura bucal satisfatória, porém limitada à direita. $\mathrm{O}$ seguimento conjunto com outras especialidades na reabilitação da paciente, juntamente com a boa funcionalidade do retalho, contribuíram para a evolução satisfatória observada no retorno ambulatorial após o sexto mês da cirurgia. 
Em relação às complicações relacionadas à técnica, estão associados relatos de necrose quando são utilizados grandes retalhos, como descrito na revisão elaborada por Nthumba ${ }^{22}$ ou em pacientes com abuso de tabaco são mais propensos a ter complicações com necrose do retalho ${ }^{10}$. As necroses ocorrem devida a perfusão insuficiente no retalho ou excessiva tensão aplicada durante $o$ procedimento. No caso da paciente desse relato, apesar do desbridamento de uma área extensa e a mesma ser fumante de longa data, não houve intercorrências no período pós-operatório. Apresentou evolução favorável com cicatrizes planas, hipocromicas, retalho com bom aspecto, abertura bucal satisfatória.

A região doadora também é motivo de preocupação pós cirúrgica, pois é primordial preservar a flexibilidade e funcionalidade dos tecidos da área em que foram removidos os retalhos. De acordo com Pallua e Noah ${ }^{19}$, a manobra de tunelamento melhora significativamente o local doador reduzindo cicatrizes. $\mathrm{O}$ ponto de pivô é na região supraclavicular e permite a utilização do retalho na parte superior do tórax, pescoço, queixo e bochecha. O emprego desta técnica nesse estudo de caso demonstrou que a região doadora se encontrava com boa cicatrização, aspecto plano sem prejuízo à mobilidade e amplitude de movimento para a paciente. Além disso, foi observado que a estética do local não sofreu prejuízo, portanto, resultados funcionais e estéticos satisfatórios.

\section{Conclusão}

A FN facial é uma infecção de progressão rápida, potencialmente fatal, desafiadora e que requer o diagnóstico e a intervenção imediatos. A doença em si e o tratamento requerido, desbridamentos cirúrgico extensos, deixam danos estéticos funcionais estigmatizantes sendo essencial a globalidade do tratamento com a cirurgia reparadora após o controle infeccioso. A reconstrução funcional e estética de regiões cervicofaciais é essencial para a qualidade de vida e contribui para 
melhora da autoestima do paciente com ganho estético e psicossocial expressivo.

O retalho supraclavicular estendido é uma excelente opção para reconstrução da face em pacientes em que foram removidas extensas áreas de pele, pois apresentaram boa vascularização e aspecto estético adequado no pósoperatório. O procedimento é vantajoso quando comparado com outros tipos de retalhos devido ao seu suprimento de sangue axial ser mais abundante e suficiente para suprir a região afetada. Soma-se ao fato, a vantagem de não exigir a presença de um cirurgião microvascular durante a realização da cirurgia nem expansores de pele. Por tratar-se de técnica de extensão de retalho sem uso de expansores, por meio da autonomização, pudemos constatar que o resultado apresentado neste caso foi semelhante aos relatados com uso deles encontrados na literatura.

\section{Referências}

1. Hunt JP, Buchmann LO. The supraclavicular artery flap for lateral skull and scalp defects: effective and efficient alternative to free tissue transfer. J Neurol Surg Rep. 2014;75(1):e5-e10.

2. Motamed S, Hormozi AJ K, Marzban S. Expanded occipito-cervico-pectoral flap for reconstruction of burned cervical contracture. Burns. 2003; 29(8):842-

844.

3. Ninkovic M, Moser-Rumer A, Ninkovic M, Spanio S, Rainer C, Gurunluoglu R. Anterior neck reconstruction with pre-expanded free groin and scapular flaps. Plast Reconstr Surg. 2004 Jan;113(1):61-8.

4. Abrantes $\mathrm{JC}$ et al. Reconstrução da região cervical e do terço médio e inferior da face com retalho livre e expandido paraescapular pós-sequela de queimadura. Rev Bras Cir Plást. 2006; 21(1): 56-60. 
5. Topalan M, Guven E, Demirtas Y. Hemifacial resurfacing with prefabricated induced expanded supraclavicular skin flap. Plast Reconstr Surg. 2010; 125(5): 1429-1438.

6. Hofer SOP, Mureau MAM. Pedicled perforator flaps in the head and neck. Clin Plast Surg. 2010; 37(4): 627-640.

7. Hormozi AK, Shafii MR. Mentosternal contracture treated with a massively expanded supraclavicular flap in a 25-year-old man: a case report. Burns. 2010; 36(6): e102-e105.

8. Colletti G, Autelitano L, Tewfik K, Rabbiosi D, Biglioli F. Autonomized flaps in secondary head and neck reconstructions. Acta Otorhinolaryngol Ital. 2012; 32(5):32935 .

9. Kokot N. et al. Use of the supraclavicular artery island flap for reconstruction of cervicofacial defects. J Otolaryngol Head Neck Surg. 2014; 150(2): 222-228.

10.Patel UA, Hartig GK, Hanasono MM, Lin DT, Richmon JD. Locoregional Flaps for Oral Cavity Reconstruction: A Review of Modern Options. Otolaryngol Head Neck Surg. 2017; 157(2):201-209.

11.ZHAO, Muxin et al. Resurfacing of the Mid and Lower Faces by Preexpanded Supraclavicular Flap. Journal of Craniofacial Surgery, v. 28, n. 6, p. 1565-1569, 2017.

12. Alves HRN. Estudo comparativo do uso do retalho fasciocutâneo supraclavicular em defeitos oncológicos nas reconstruções de cabeça e pescoço imediata e de resgate [tesis]. São Paulo: Faculdade de Medicina; 2018.

13.Li Y, Zhao Z, Wu D, Li H, Guo Z, Liu X. Clinical application of supraclavicular flap for head and neck reconstruction. Eur Arch Otorhinolaryngol. 2019; 276(8):2319-2324.

14.HERNANDEZ DA. et al. Facial necrotizing fasciitis in adults: a systematic review. Heighp. Otol. Rhinol. 2017; 1(1): 20-31.

15. Neto NT et al. Infecções graves de partes moles: relato de caso de fasciíte necrotizante de face utilizando curativo a vácuo e revisão da literatura. Rev Bras Cirur Plást. 2011; 26(2):352-359.

16.Pallua N, Machens HG, Rennekampff O, Becker M, Berger A. The fasciocutaneous supraclavicular artery island flap for releasing postburn mentosternal contractures. Plast Reconstr Surg. 1997; 99(7):1878-84; discussion 1885-6.

17.Trautman J, Gore S, Potter M, Clark J, Hyam D, Tan NC, Ngo Q, Ashford B. Supraclavicular flap repair in the free flap era. ANZ J Surg. 2018; 88(6):540-546. 
18. Bhaya MH., Har-el G. Resident training in head and neck flap reconstruction in US academic otolaryngology programmes. J. Lar. Otol., Lond. 2001; 115(2):119.

19.Pallua N, Noah EM. The tunneled supraclavicular island flap: an optimized technique for head and neck reconstruction. Plast Reconstr Surg. 2000; 105(3):842-851.

20.Spence RJ. An algorithm for total and subtotal facial reconstruction using an expanded transposition flap: a 20-year experience. Plast Reconstr Surg. 2008; 121(3):795-805.

21.Yang Z, Hu C, Li Y, Tang Y, Zhao M, Chen W, Xu L, Liu Y. Pre-expanded cervicoacromial fasciocutaneous flap based on the supraclavicular artery for resurfacing post-burn neck scar contractures. Ann Plast Surg. 2014 Sep;73 Suppl 1:S92-8.

22.Nthumba PM. The supraclavicular artery flap: a versatile flap for neck and orofacial reconstruction. J Oral Maxillofac Surg. 2012; 70(8):1997-2004.

23.Pallua N, Kim BS. Pre-expanded supraclavicular artery perforator flap. Clin Plast Surg. 2017; 44(1):49-63.

\section{How to cite this article (APA format):}

Rocha, K.D.L.S.; Carvalho, V.H.M.; Cidade, P.I.M.; Macedo, A.S.S.; Furtado, M.I.F.P.; Vieira, L.C.B.B.; Rolim, F.F.A. (2021). Facial reconstruction with extended supraclavicular flap after necrotizing fasciitis: Case report. Am. In. Mult. J., Oct. (11) 6, 43-59.

Received: 10/18/2021

Accepted: 10/24/2021

Published: 10/31/2021 